\title{
Automated detection of vehicles license plate using image processing techniques
}

\author{
Farhana Ahmad Poad ${ }^{1}$, Noor Shuraya Othman ${ }^{2}$, Roshayati Yahya@ Atan ${ }^{3}$, Jusrorizal Fadly Jusoh ${ }^{4}$, \\ Mumtaz Anwar Hussin ${ }^{5}$ \\ ${ }^{1,2,4}$ Department of Communication Engineering, Universiti Tun Hussein Onn Malaysia (UTHM), Malaysia \\ ${ }^{3}$ Jabatan Teknologi Maklumat dan Konunikasi, Politeknik Sultan Abdul Halim Muadzam Shah, Malaysia
}

\begin{tabular}{l} 
Article Info \\
\hline Article history: \\
Received Aug 28, 2019 \\
Revised Oct 30, 2019 \\
Accepted Nov 13, 2019 \\
\hline
\end{tabular}

Keywords:

ADLP

OCR

Split and merge segmentation

\begin{abstract}
The aim of this project is to design an Automated Detection of License Plate (ADLP) system based on image processing techniques. There are two techniques that are commonly used in detecting the target, which are the Optical Character Recognition (OCR) and the split and merge segmentation. Basically, the OCR technique performs the operation using individual character of the license plate with alphanumeri characteristic. While, the split and merge segmentation technique split the image of captured plate into a region of interest. These two techniques are utilized and implemented using MATLAB software and the performance of detection is tested on the image and a comparison is done between both techniques. The results show that both techniques can perform well for license plate with some error.
\end{abstract}

Copyright () 2020 Institute of Advanced Engineering and Science. All rights reserved.

\section{Corresponding Author:}

Farhana binti Ahmad Po'ad,

Department of Communication Engineering,

Universiti Tun Hussein Onn Malaysia (UTHM), Malaysia.

Email: farhana@uthm.edu.my

\section{INTRODUCTION}

Nowadays as the lifestyle are keep on improving, the number of cars in Malaysia are increasing rapidly and become as an important facilities for the modern citizen to go to work, shopping, lectures and etc. In line with the increase in the number of vehicles in Malaysia, issues such as traffic jams and lack of parking spaces are increasing with less action to control the situation. As comparing between the number of car and number of parking space available, it indicates that there is lack of parking space [1]. This situation becomes more difficult in urban areas with high numbers of population. In the situation of no parking space available, some driver will do double parking as shown in Figure 1. The figure shows that the red car is parked outside the allocated parking space and blocking the blue car that park in the given parking space.

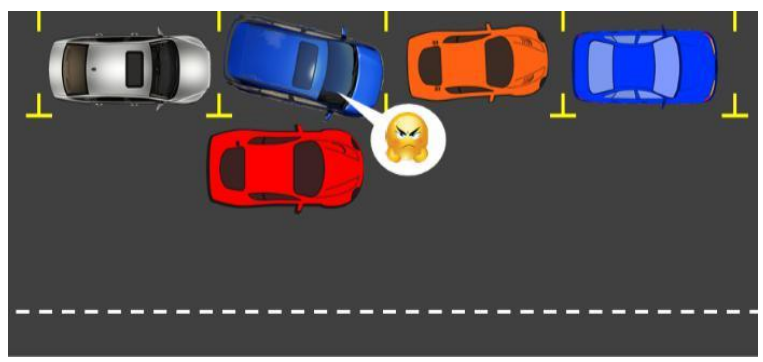

Figure 1. Illustration of double parking situation 
In addition, the double parking can cause traffic jam and other traffic offense that might have implications on road users. Initially the road allows two cars to pass through at a time. However, with double parking, the road can only fit one car at a time. This reduces the traffic flow and causing the traffic jam. Therefore, a hybrid system known as Automated Detection of License Plate (ADLP) is proposed which uses Optical Character Recognition (OCR) and split and merge segmentation techniques to determine the individual character of number of the detected car, which is parked at the illegal area. Generally, the ADLP system takes input from the plate number by using cameras and reconise the alphanumeric characters using image processing technique. This technique is typically used in transportation scope in order to enhance the safety and mobility and also the productivity of advanced technologies [2, 3]. Basically, the OCR technique uses each character of the license plate and only suitable for alphanumeric. While the split and merge segmentation technique, split the area of image into a smaller area before merged it into a specific area of interest. These techniques are commonly used in toll payments [4], parking management [5, 6], road-traffic monitoring [7], security [8], crime identification [9] and etc [10].

\section{LITERATURE REVIEWS}

Qadri et. Al [11] propose a system that able to detect a vehicle and capture the image of vehicle by using segmentation and OCR techniques to trace the plate number and to identify the character. According to [11], the system is able to detect the plate number robustly without mentioning its accuracy. In addition, Rathore et. Al [5] propose a system using the same techniques with $99 \%$ accuracy of detection when the images are captured at fixed position that directly facing to the camera. Meanwhile, a research done by the S. Bhatkar et.al [12] develop a number plate detection system using Sobel Edge detection method. The system is successfully detected the edges and fills the holes with less than 8 pixels. This method is efficient due to less time consuming. Bhat et. Al [13] mention in their work that Sobel Edge method and morphological operation are able to predict the number of license plate in various shapes, sizes and different background colour.

Zhang et.al [14] propose a work entitled license plate recognition based on MATLAB by using gray-scale tensile, Log-Prewitt operator and self-adaptive median filter 7 techniques. The Log-Prewitt operator as mentioned in [13] is better than the Sobel method, while the self-adaptive median filter is the most practical methods to eliminate noise. Besides that, Ranglani et. Al [15] propose a technique consists of four levels of implementation, which are mathematical technique, thresholding (Otsu method), normalization (Hough transform) and boundary regional features and segmentation part. These 4 recognition levels use the template matching to recognize the characters of the license plate. The system is successfully function as required with $83.5 \%$ accuracy.

In this work, a hybrid technique that consists of OCR and split and merge segmentation techniques [16] are used to detect the number of license plate. The proposed technique is similar to the previous work done by [10], however, this work analysed the license plate in a few situations such as the distance and the angles of the license plate. Both techniques are widely used in the previous research because of the accuracy of the results produced. Basically, the OCR technique can function by itself but then it will encounter several problems that could be able to tackle by using split and obtained the accurate and successful results [17].

\section{PROPOSED METHOD}

This section reviews on the image processing methods that can be implemented for the detection of license plate which consists of OCR and split and merge techniques.

\subsection{Optical Characters Recognition (OCR)}

The OCR is an electronic process of changing of images into machine-encoded information. This is a general procedure to exchange the printed text into digital which allow the information to be edited, stored and displayed online [18]. Each of the characters obtained from plate number having the same size with the character save on database. The OCR technique uses template-matching algorithm to reconise each character. The character image is later compared with the database template save in the memory and the similarity of both information is measured $[19,20]$.

The similarity is measured using statistical method (correlation coefficient) and the most similar character is obtained before the number is predicted. The correlation coefficient is measure between a numbers of known images with the same size of unknown images to find the best match of image based the highest correlation coefficient value. There are 2 types of correlations, which are Auto-Correlation Function (ACF) and Cross-Correlation Function (CCF). The ACF involves only one signal and provides information of the signal in the time domain. However, the CCF measures the similarities between two signals. In this 
work, there are 2 signal which are unknown input image and known template image, therefore the cross-correlation is used to measure the similarities of two signals.

Let $F 1(j, k)$ and $F 2(j, k)$ for $1 \leq j \leq J$ and $1 \leq k \leq K$ represent two discrete image denoting the image to be searched and the template respectively. The normalized cross-correlation between the image pair is defined as for $\mathrm{m}=1,2, \ldots \mathrm{M}$ and $\mathrm{n}=1,2, \ldots \mathrm{N}$, where $\mathrm{M}$ and $\mathrm{N}$ are odd integers.

$$
R(m, n)=\frac{\sum_{j} \sum_{k} F_{1(j, k)} F_{2\left(j-m+\frac{M+1}{2}, k-n+\frac{N+1}{2}\right)}}{\left[\sum_{j} \sum_{k}\left|F_{1(j, k)}\right|^{2}\right]^{1 / 2}\left[\sum_{j} \sum_{k}\left|F_{1(j, k)} F_{2\left(j-m+\frac{M+1}{2}, k-n+\frac{N+1}{2}\right)}\right|^{2}\right]^{1 / 2}}
$$

According to [18], there might be some error during the recognition due to the similarities of some characters such as $\mathrm{B}$ and $8, \mathrm{E}$ and $\mathrm{F}, \mathrm{D}$ and $\mathrm{O}, \mathrm{S}$ and $5, \mathrm{Z}$ and 2.

\subsection{Split and Merge Segmentation}

Split and merge segmentation technique aims to partition a digital image into multiple region to restructure image representation into a meaningful information which is easy to analyse. The image area is divided into smaller uniform areas and these smaller areas are again combined to indicate the area of interest [21-23]. There are 4 steps involve in split and merge algorithm which are (a) Divide any area Ri into four equal areas, (b) Divide the area continously until there is no area left, (c) combine any two areas $\mathrm{Ri}$ and $\mathrm{Rj}$ for which $\mathrm{P}(\mathrm{Ri} \cup \mathrm{Rj})=\mathrm{TRUE}$. Then (d) continue combining until there is no area left for combining [19].

The major disadvantages of split and merge algorithm is lacking of instruction on the selection of features, highly depending on limit values for both splitting and merging, as well as requires a large number of computations. However, by combining these two techniques, the system is able to minimize the disadvantages of each technique. Besides that, these techniques are much simpler than other methods and easy to comprehend.

\section{PROPOSED ADLP SYSTEM}

Figure 2 shows the block diagram of the proposed ADLP system, whereby the input image is captured using a camera based on Red, Green and Blue (RGB) format. The quality of the camera should be high in order to get the proper recognition of characters. Next, the license plate is obtained from the original image and focussing on the plate number. After completing the segmentation stage, the characters is detected using the template matching in OCR technique.

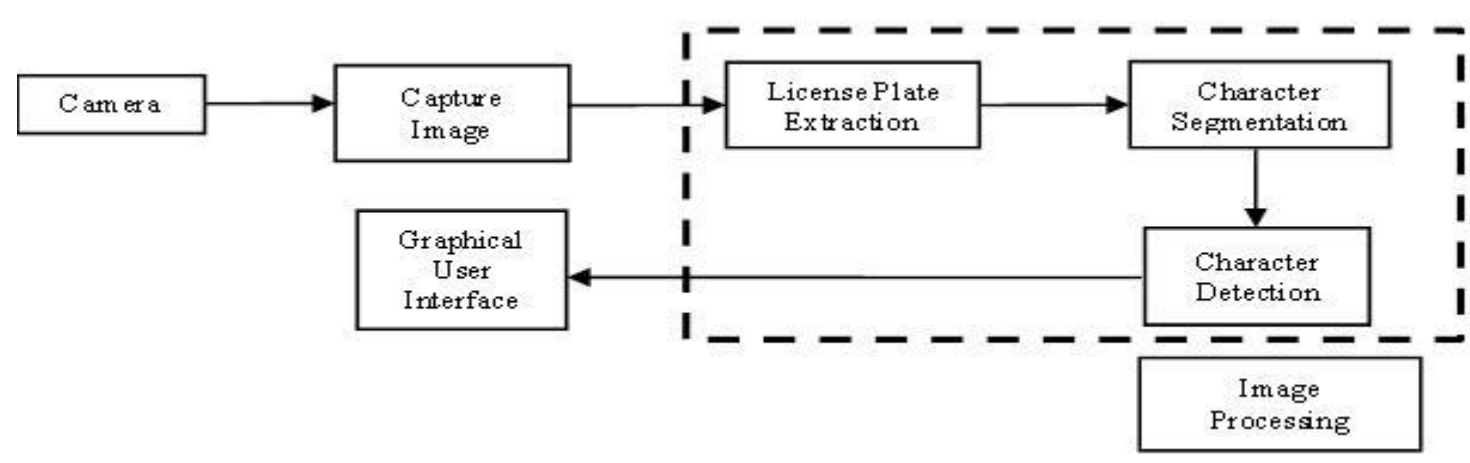

Figure 2. Block diagram of the proposed ADLP system

Figure 3 shows the flowchart of proposed ADLP system that is implemented in this work. The system starts with image acquisition, where a digital image is obtained through a digital camera or webcam. The system will be activated only if it is detected a car parking illegally at 'NO PARKING' zone or at the disable parking area. The captured image is later converted the existing RGB image into gray scale format using MATLAB software. In preprocessing stage, the noise on the image is eliminated, while the contrast or the features of the image is enhanced. The techniques involve in the preprocessing stage are gray processing, median filtering and license plate detection/extraction. Once the input image detection completed, the image is identify before the license plate can be extracted from its original image. In the 
character detection and recognition stage, the license plate area obtained will be processed and divided into the smallest area in order to segmentise each of the characters. Finally, the output of character, which refers to the license plate number, will be shown on the Graphical User Interface (GUI) for user references.

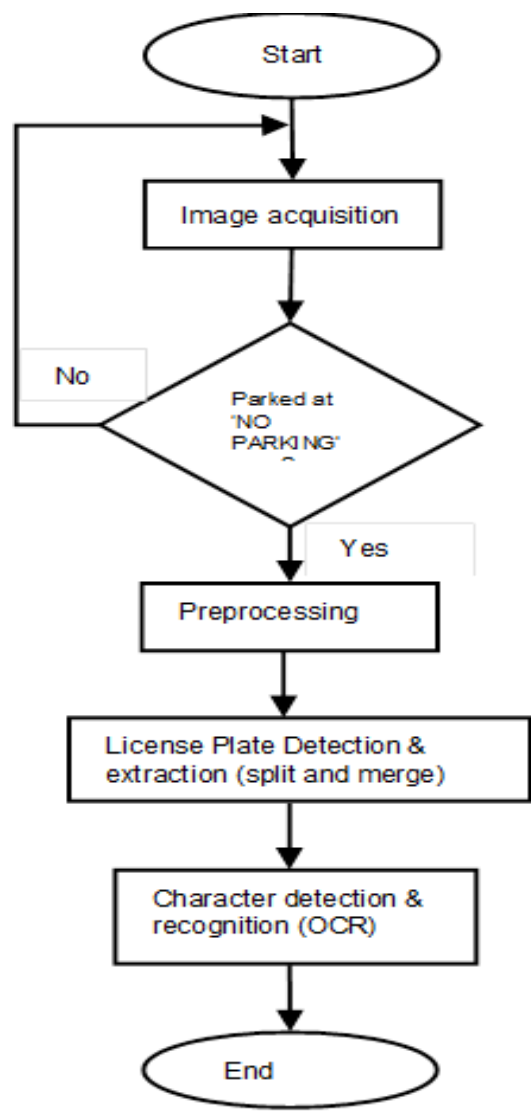

Figure 3. Flowchart of the proposed ADLP system

Since the proposed system is a combination of both techniques, the hybrid process is happened at the license plate detection and extraction steps, once the license plate is detected and extracted from the original image, the merge and split segmentation techniques triggered to merge and split them into a small region. The system continued detected each character on it then using the template matching in OCR techniques to recognize the characters on the license plate.

\section{RESULTS AND ANALYSIS}

\subsection{Optical Characters Recognition (OCR)}

Table 1 shows the results obtained using Optical Character Recognition (OCR) technique. From seven images, five images are from the license plates that are attached with the car body while the other two images are entirely the license plates. The system managed to detect the characters successfully except for the fifth images due to the darker image, which causes the characters are unidentified. While for the image of license plate only, the system is unable to recognize all the characters due to the character might be not matched with the template. Therefore, the recognition depends on the input image quality.

\subsection{Split and Merge Segmentation}

Table 2 shows the results obtained using split and merges segmentation technique. According to the results, the detection of license plate number is greatly affected by the distance between camera and target of the input image. If the input is at a certain distance from the camera and the fuzzy image is captured, therefore, the characters are difficult to be segmentised. 


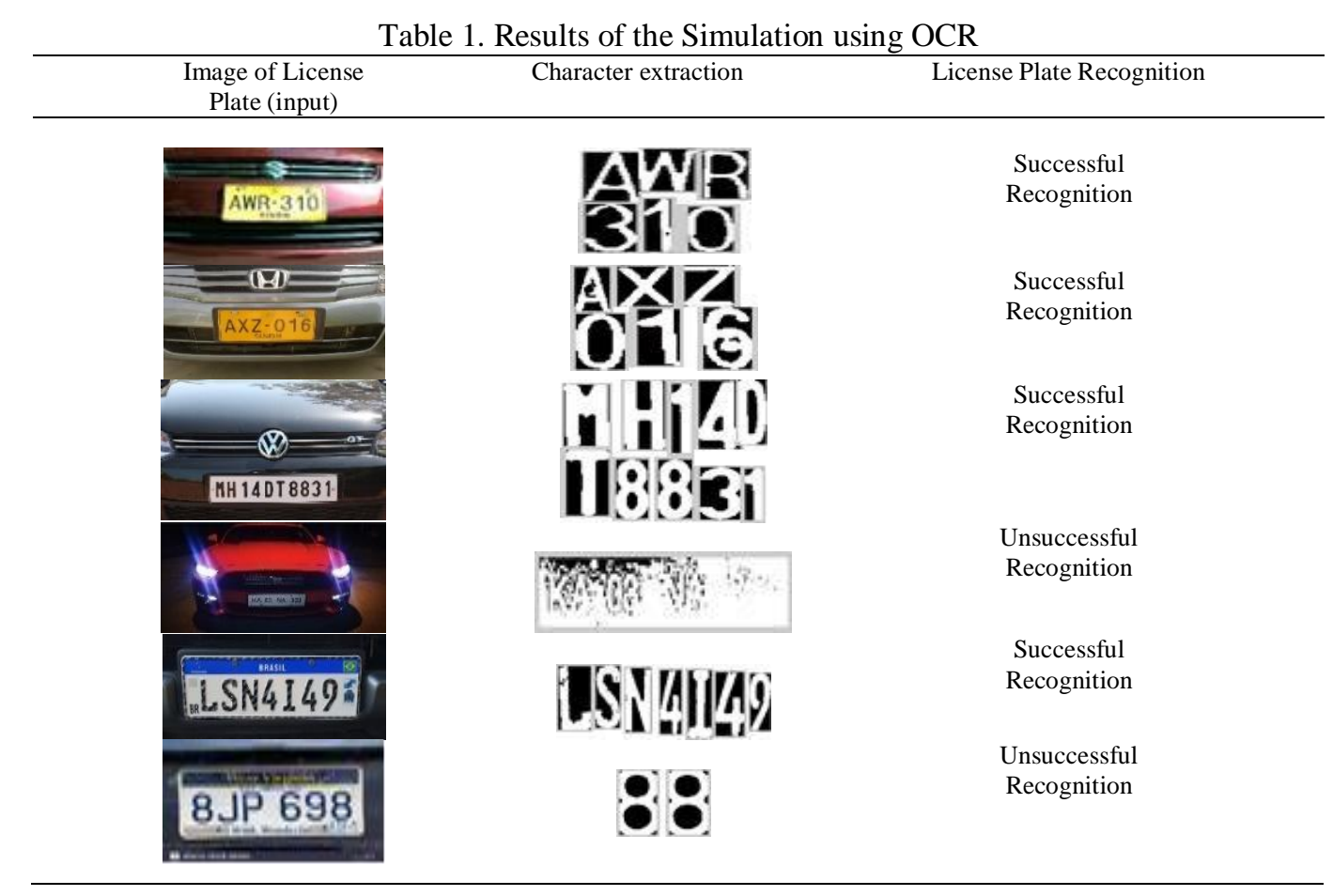

Table 2. Result of the Simulation using Split and Merge

\begin{tabular}{cc}
\hline Input Image & Output Image \\
\hline
\end{tabular}

\subsection{Hybrid OCR and Split and Merge Segmentation}

The results are analyzed based on the distance and the angle of the license plate [24]. Different license plate from above results has been used to show the relation of the factors with this technique. Table 3 shows the simulation results from the images of the license plate that are taken at various distances. The readings are observed for 10 times at each distance between $20 \mathrm{~cm}$ to $100 \mathrm{~cm}$. This analysis is performed 
to determine the effectiveness of the proposed technique while detecting the license plate at different distances. From the results obtained, it can be seen that the system only capable to detect the license plate at the distance below $40 \mathrm{~cm}$, however the characters are not accurately recognized. The detection is unsuccessful for the distance more than $40 \mathrm{~cm}$ due to the fuzzy image and characters are unable to be segmented.

Table 3. Simulation Results of the Proposed Technique based on Distance

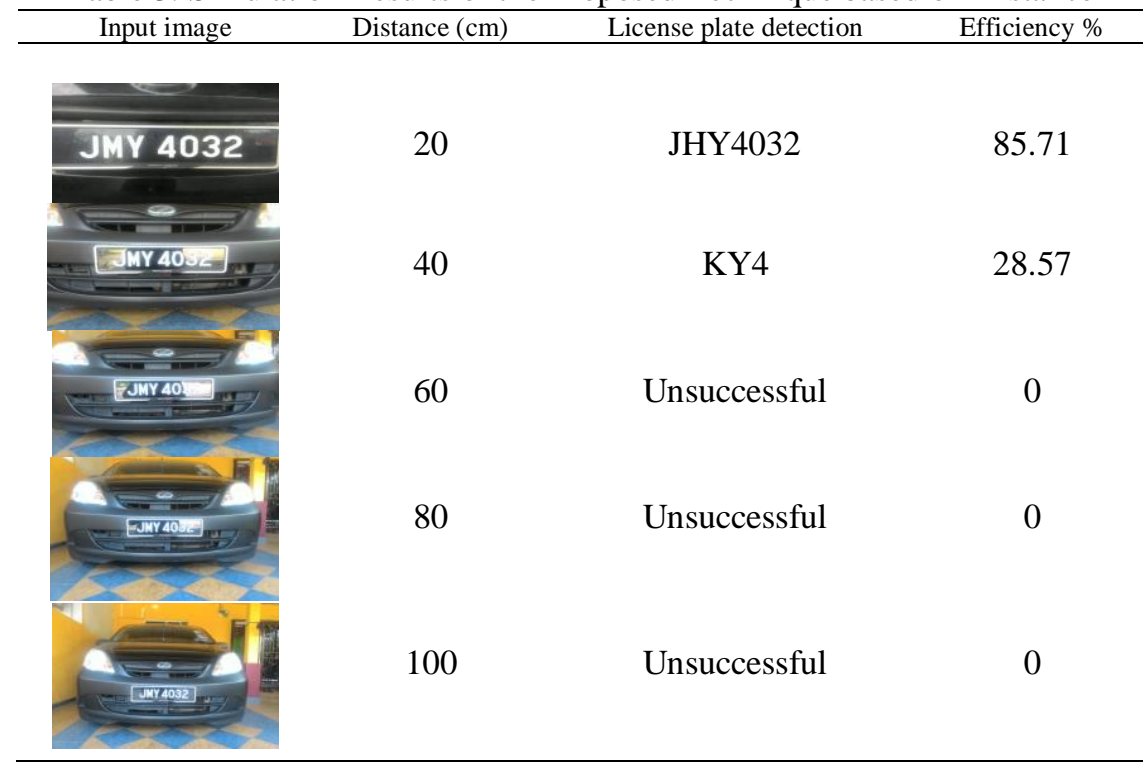

The system is later observed in terms of five different angles of captured image as shown in Table 4. The images are captured at the angles of $22^{\circ}, 45^{\circ}, 90^{\circ}, 135^{\circ}$ and $157^{\circ}$ degrees. The readings are taken more than 10 times at each angle. The results show the system is able to detect the license plate number only at $45^{\circ}, 90^{\circ}$ and $135^{\circ}$ degrees. However, the detected license plate numbers are not accurate due to the character not segmented well, which leads to the system displays wrong characters on the screen panel. While the images taken at $22^{\circ}$ and $157^{\circ}$ degress, are unable to detect the license plate due to the regions of image were failed to fill during extraction process.

Table 4. Simulation Results of the Proposed Technique based on Angle

\begin{tabular}{|c|c|c|c|}
\hline Input Image & Angle $\left({ }^{\circ}\right)$ & License plate detection & Efficiency $\%$ \\
\hline & 22 & Unsuccessful & 0 \\
\hline JOF 8500 & 45 & Y4 & 28.57 \\
\hline JOF 8503 & 90 & JQF8A & 57.14 \\
\hline Jif 8503 & 135 & QF85 & 57.14 \\
\hline & 157 & Unsuccessful & 0 \\
\hline
\end{tabular}




\section{CONCLUSION AND RECOMMENDATIONS}

As the conclusion, the Automated Detection of License Plate (ADLP) system is developed to detect Malaysia license plate number using hybrid OCR and split and merge segmentation techniques. The performance of each technique has been analysed and a hybrid technique has been proposed which merge both techniques to get better performance. The system is considered successful to recognize and detect the input images, however there are some difficulties occur during the simulation stage whereby the proposed system is unable to detect the input due to certain reason such as the character not segmented well and the regions of image were failed to fill during extraction process. For instant, when the image of license plate number is fuzzy, the characters sizes on license plate number are small which causes an error during processing stage. The distance between camera and plate number also plays an important role to reduce the possibilities of error during processing stage. Future works can be done to detect the license plate number during nighttime [25] or raining day [26]. From the field test done, the system is unable to detect the input image in dark condition; therefore an improvement is needed on this factor, which could increase the efficiency of the system in future. A high-resolution camera also needed to produce a better image sample. Finally, by using the proposed hybrid technique, it is expected that the system is able to produce more accurate result for license plate number detection.

\section{ACKNOWLEDGEMENTS}

The authors would like to thank Research Management Center (RMC) Universiti Tun Hussein Onn Malaysia (U852, H180 Tier 1 Fund and H303 GPPS Fund) for sponsoring the research and development of this project.

\section{REFERENCES}

[1] A. Ahmad, "parking-woes-our-towns-and-cities," New Straits Times, 2017. [Online]. Available: https://www.nst.com.my/cbt/2017/10/293703/parking-woes-our-towns-and-cities.

[2] C. N. E. Anagnostopoulos, "License plate recognition: A brief tutorial," IEEE Intell. Transp. Syst. Mag., vol. 6, no. 1, pp. 59-67, 2014.

[3] I. Chibuisi and O. M. C, “MATLAB Based Vehicle Number Plate Recognition,” vol. 3, no. Iv, pp. 353-357, 2015.

[4] S. Sagar, J. Jori, A. Kale, K. Khodade, and P. Mali, "Vehicle Counting and Automated Toll Collection System using Image Processing,” IJCSN Int. J. Comput. Sci. Netw., vol. 5, no. 2, 2016.

[5] M. Rathore and S. Kumari, "Tracking Number Plate From Vehicle Using Matlab," Int. J. Found. Comput. Sci. Technol., vol. 4, no. 3, pp. 43-53, 2014.

[6] D. Sathiyanarayanan, S. Shrihari, and A. Veeramuthu, "A novel methodology for vehicle plate localization, segmentation, and recognition for real scenario using algorithms," 2015 Int. Conf. Commun. Signal Process. ICCSP 2015, pp. 1593-1597, 2015.

[7] R. R. Raskar and P. R. G. Dabhade, "Automatic Number Plate Recognition ( ANPR )," vol. 5, no. 3, pp. 547-550, 2015.

[8] S. M. Malik, M. A. Iqbal, Z. Hassan, T. Tauqeer, R. Hafiz, and U. Nasir, "Automated over speeding detection and reporting system,” 16th Int. Power Electron. Motion Control Conf. Expo. PEMC 2014, pp. 1104-1109, 2014.

[9] N. Shirodkar and P. Uchil, "Number Plate Detection using Image Processing for Automated Toll Collection to prevent fraudulent behaviour," vol. 4, no. 5, pp. 1922-1927, 2015.

[10] S. Rani, "A Review of Recognition Technique Used Automatic License Plate Recognition A Review of Recognition Technique used Automatic License Plate Recognition System," no. September, 2015.

[11] M. T. Qadri and M. Asif, "Automatic number plate recognition system for vehicle identification using optical character recognition,” 2009 Int. Conf. Educ. Technol. Comput. ICETC 2009, pp. 335-338, 2009.

[12] S. Bhatkar, J. Chavda, and S. Lad, "Indian Vehicle Number Plate Detection Using Image Processing.," pp. 1825-1831, 2017.

[13] R. Bhat and B. Mehandia, "Recognition of Vehicle Number Plate Using Matlab," Int. J. Innov. Res. Electr. Electron. Instrum. Control Eng., vol. 2, no. 8, pp. 2321-2004, 2014.

[14] X. Zhang, F. Xu, and Y. Su, "Research on the license plate recognition based on MATLAB," Procedia Eng., vol. 15, pp. 1330-1334, 2011.

[15] J. Ranglani and V. Lachwani, “Automatic number plate recognition,” Wikipedia, vol. 2, no. 3, pp. 1-18, 2012.

[16] A. Goyal and R. Bhatia, "Various Techniques for Number Plate Recognition-A Review," Int. J. Comput. Appl., vol. 143, no. 11, pp. 975-8887, 2016.

[17] C. S. Yang and Y. H. Yang, "Improved local binary pattern for real scene optical character recognition," Pattern Recognit. Lett., vol. 100, pp. 14-21, 2017.

[18] L. Eikvil, "Optical Character Recognition,” no. December, 1993.

[19] S. Ozbay and E. Ercelebi, “Automatic Vehicle Identification by Plate Recognition,” pp. 222-225, 2005.

[20] I. Kastelan, S. Kukolj, V. Pekovic, V. Marinkovic, and Z. Marceta, "Extraction of text on TV screen using optical character recognition,” 2012 IEEE 10th Jubil. Int. Symp. Intell. Syst. Informatics, SISY 2012, pp. 153-156, 2012.

[21] "Split and Merge: A Region Based Image Segmentation," vol. 9359, no. 8, pp. 306-309, 2017. 
[22] M. S. Farag, M. M. M. El Din, and H. A. El Shenbary, "Parking entrance control using license plate detection and recognition," vol. 15, no. 1, pp. 476-483, 2019.

[23] Z. Karim, N. R. Paiker, M. A. Ali, G. Sorwar, and M. M. Islam, "Pattern based object segmentation using split and merge,” IEEE Int. Conf. Fuzzy Syst., pp. 2166-2169, 2009.

[24] A. K. Parida, S. H. Mayuri, P. Nayk and N. Bharti, "Number Plate Recognition System for Vehicle using MATLAB," International Research Journal of Engineering and Technology (IRJET), pp. 846-851, 2016.

[25] FengWanli and GaoShangbing, "A Vehicle License Plate Recognition Algorithm in Night Based on HSV," 2010 3rd International Conference on Advanced Computer Theory and Engineering(ICACTE), Chengdu, pp. V4-53-V4-56, 2010.

[26] S. Yoshimori, Y. Mitsukura, M. Fukumi, N. Akamatsu and R. Khosal, "License plate detection system in rainy days," Proceedings 2003 IEEE International Symposium on Computational Intelligence in Robotics and Automation. Computational Intelligence in Robotics and Automation for the New Millennium (Cat. No.03EX694), Kobe, Japan, pp. 972-976 vol.2, 2003.

\section{BIOGRAPHIES OF AUTHORS}

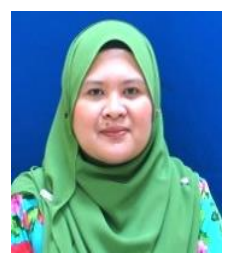

Farhana Ahmad Poad is a Senior Lecturer at Universiti Tun Hussein Onn Malaysia (UTHM) and senior researcher for Wireless and Radio Science Center (WARAS), UTHM. She received her degree in B.Sc. of Electrical \& Electronics Engineering (Hons) from Kolej Universiti Teknologi Tun Hussein Onn Malaysia (KUiTTHO) in 2003 and Master Degree in Electrical Engineering from Universiti Tun Hussein On Malaysia (UTHM) in 2007. She received PhD in 2016 specialization in Communication System Engineering from Universiti Sains Malaysia (USM). She served Universiti Tun Hussein Onn Malaysia (UTHM) as a tutor from year 2003 until 2006 and as a lecturer in the year 2007 until today. Her main areas of research are Electromagnetic Compatibility (EMC), Wireless System Design and Radio Frequency Identification (RFID). A researcher as a member of Radio Frequency Identification (RFID) group at the Auto-ID Laboratory at USM. Her research interest is in the areas of RFID, wireless sensor networking and antenna.

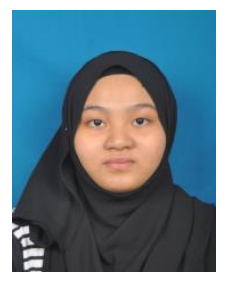

Noor Shuraya Othman currently does her degree in Electronic engineering at Universiti Tun Hussein Onn Malaysia (UTHM). Her final year project is about the automated detection of license plate using the image processing.

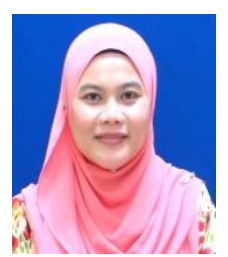

Roshayati Yahya@Atan received her first degree in Universiti Tun Hussein Onn Malaysia (UTHM) in Electrical and Electronics Engineering (Telecommunication) in 2006. She joined UTHM in 2007 as a tutor and honored a Master degree in Electrical and ElectronicsTelecommunications from Universiti Teknologi Malaysia (UTM) in 2009. She awarded a PhD in the same university as she was doing her M.Eng at Wireless Communication Centre (WCC), UTM. Her research interests including antenna design, RF and microwave, and antenna for biomedical engineering applications.

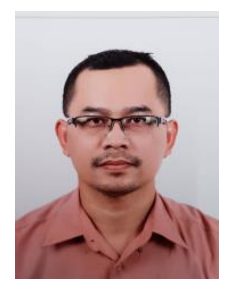

Ts. Jusrorizal Fadly Jusoh is a Senior Lecturer at Information Technology \& Communication Department, Politeknik Sultan Abdul Halim Mu'adzam Shah (POLIMAS). He received a Diploma in Computer Science (Information Technology) from UTM, Kuala Lumpur in 2000. He Received his Bachelor's Degree in Information Technology (Networking) from UUM in 2003 and a Master Degree in Technical and Vocational Education from KUiTTHO in 2005. Membership of Malaysian Board of Technologists (MBOT) since 2018 and member of Internet Society (ISOC) since 2015. Thirteen years of teaching experience in a polytechnic system involves computer hardware, database application, open source operating system, computer networking, network security and secure mobile computing.

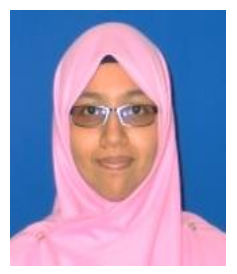

Mumtaz Anwar Hussin received her Bachelor Degree of Electronics Engineering (Hons) from UTHM in 2018. Currently, she is pursuing her Master Degree in Communication Engineering at UTHM. Her researches of interests are focussing on Wireless Sensor Network (WSN), Internet of Things (IoT) and image processing. Presently, she is working on image compression by using hybrid method for image transmission over ZigBee network 